\title{
BMJ Open Socioeconomic inequalities in the prevalence of complex multimorbidity in a Norwegian population: findings from the cross-sectional HUNT Study
}

\author{
Kristin Hestmann Vinjerui (D , ,,2 Ottar Bjerkeset, ${ }^{3,4}$ Johan H Bjorngaard, ${ }^{3,5}$ \\ Steinar Krokstad, ${ }^{1,2}$ Kirsty A Douglas, ${ }^{6}$ Erik R Sund ${ }^{1,3,7}$
}

To cite: Vinjerui $\mathrm{KH}$, Bjerkeset 0 , Bjorngaard $\mathrm{JH}$, et al. Socioeconomic inequalities in the prevalence of complex multimorbidity in a Norwegian population: findings from the cross-sectional HUNT Study. BMJ Open 2020;10:e036851. doi:10.1136/ bmjopen-2020-036851

- Prepublication history and additional material for this paper are available online. To view these files, please visit the journal online (http://dx.doi. org/10.1136/bmjopen-2020036851).

Received 07 January 2020 Revised 31 March 2020 Accepted 12 May 2020

Check for updates

(C) Author(s) (or their employer(s)) 2020. Re-use permitted under CC BY-NC. No commercial re-use. See rights and permissions. Published by BMJ.

For numbered affiliations see end of article.

Correspondence to Dr Kristin Hestmann Vinjerui; kristin.vinjerui@ntnu.no

\section{ABSTRACT}

Objectives Multimorbidity, the co-occurrence of multiple long-term conditions, is common and increasing. Definitions and assessment methods vary, yielding differences in estimates of prevalence and multimorbidity severity. Sociodemographic characteristics are associated with complicating factors of multimorbidity. We aimed to investigate the prevalence of complex multimorbidity by sex and occupational groups throughout adulthood. Design Cross-sectional study.

Setting The third total county survey of The NordTrøndelag Health Study (HUNT), 2006-2008, Norway. Participants Individuals aged 25-100 years with classifiable occupational data and complete questionnaires and measurements.

Outcome measure Complex multimorbidity defined as 'the co-occurrence of three or more chronic conditions affecting three or more different body (organ) systems within one person without defining an index chronic condition'.

Analysis Logistic regression models with age and occupational group were specified for each sex separately. Results 38027 of 41193 adults (55\% women) were included in our analyses. $54 \%$ of the participants were identified as having complex multimorbidity. Prevalence differences in percentage points ( $\mathrm{pp}$ ) of those in the low occupational group (vs the high occupational group (reference)) were $19(95 \% \mathrm{Cl}, 16$ to 21) $\mathrm{pp}$ in women and 10 (8 to 13) pp in men at 30 years; 12 (10 to 14) pp in women and 13 (11 to 15) pp in men at 55 years; and $2(-1$ to 4) pp in women and 7 (4 to 10) pp in men at 75 years. Conclusion Complex multimorbidity is common from early adulthood, and social inequalities persist until 75 years in women and 90 years in men in the general population. These findings have policy implications for public health as well as healthcare, organisation, treatment, education and research, as complex multimorbidity breaks with the specialised, fragmented paradigm dominating medicine today.

\section{INTRODUCTION}

Multimorbidity, the co-occurrence of multiple long-term conditions in which none holds priority, ${ }^{1}$ is common and increasing. ${ }^{23}$ It challenges the individual's ability to self-manage ${ }^{45}$
Strengths and limitations of this study

- As a large, entire-county, general population health survey with a vast number of variables, the HUNT Study is ideal to estimate the prevalence of multimorbidity by self-reports and clinical measurements.

- Complex multimorbidity operationalised as three or more organ systems affected is relevant in both clinic and research, with high specificity into old age, implicating the need for coordinated multidisciplinary care and increasing comparability between studies.

- Socioeconomic position operationalised as occupations allocated in the European Socio-economic Classification scheme makes international comparison of gradients possible.

- Non-participants have lower socioeconomic position and higher mortality, thus the social gradients in prevalence of complex multimorbidity detected are likely conservative.

- The original data lacked information of chronicity of a majority of the conditions, which may lead to overestimation of complex multimorbidity.

as well as clinical decision-making ${ }^{5-7}$ due to complexity that conflicts with subspecialised medicine and clinical guidelines. Multimorbidity is associated with high healthcare utilisation in both primary and specialist care, ${ }^{8}$ including emergency department visits. ${ }^{9}$

Multimorbidity is heterogeneous, and a mere count of conditions may not imply complexity, ${ }^{15}$ requiring coordinated multidisciplinary care. In attempts to detect individuals with high needs, guidelines by and large are focused on combinations of conditions, such as concurrent mental and somatic conditions ${ }^{5} 1011$ or three or more conditions in separate organ systems, ${ }^{512}$ and consequences thereof, such as polypharmacy ${ }^{51011}$ and requirements for assistance in daily living. ${ }^{5} 10$ Individual factors that increase patient complexity include 
sociodemographic characteristics, ${ }^{13}$ social resources, ${ }^{13}$ and health and social experiences. ${ }^{13}$ Recent recommendations on multimorbidity care have taken into account social networks, ${ }^{11}$ socioeconomic positions ${ }^{11}$ and patient experiences, such as treatment burden. ${ }^{1011}$

Research results from cross-sectional studies on multimorbidity prevalence have been difficult to compare because of differences in definitions, methods, and the number and types of conditions included. ${ }^{14}{ }^{15}$ Still, associations with lower socioeconomic position, ${ }^{3}{ }^{14} 16$ female $\operatorname{sex}^{31416}$ and increasing age ${ }^{31416}$ persist across studies. Further, defining multimorbidity as simultaneously having three or more conditions increases the specificity of the multimorbidity measure into older age groups, ${ }^{1215}$ and comparability between studies increases when multimorbidity is operationalised as multiple organ systems affected. $^{12}$

Inequalities in health according to socioeconomic position are persistent, ${ }^{17}$ even in comparatively egalitarian Nordic societies. ${ }^{18}$ The association of socioeconomic differences with the occurrence of multimorbidity has been explored using multiple measures, such as education, ${ }^{14}{ }^{19}$ income $^{19}{ }^{19}$ occupation $^{3}$ and deprivation indexes. ${ }^{14} 16$ In fact, any measure of socioeconomic position will detect health differences in descriptive studies, if differences exist. ${ }^{20}$ Using an occupational classification may reflect specific work-related exposures in addition to general associations to income, material resources and social status. ${ }^{20}$

In sum, multimorbidity represents a challenge both for the individual and clinician, as well as for the coordination of healthcare. Previous multimorbidity prevalence research suggests that demographic and socioeconomic gradients operate. In Norway, multimorbidity prevalence and patterns have been partly explored. ${ }^{21}$ Studies on complex multimorbidity is lacking, and no studies have investigated sociodemographic differences. Such data can strengthen healthcare planning and clinical management of multimorbidity, as well as guide public health interventions.

Our aim is to add to former knowledge by assessing the prevalence of complex multimorbidity, defined as three or more conditions in separate organ systems, by age, sex and occupational groups, in a general population health survey.

\section{METHODS}

\section{Reporting statement}

The Strengthening the Reporting of Observational Studies in Epidemiology (STROBE) cross-sectional reporting guidelines ${ }^{22}$ were used for reporting this observational study.

\section{Study population}

The HUNT Study is a population-based health study for all adults 20 years and older living in Nord-Trøndelag County, Norway. Four surveys have been completed since

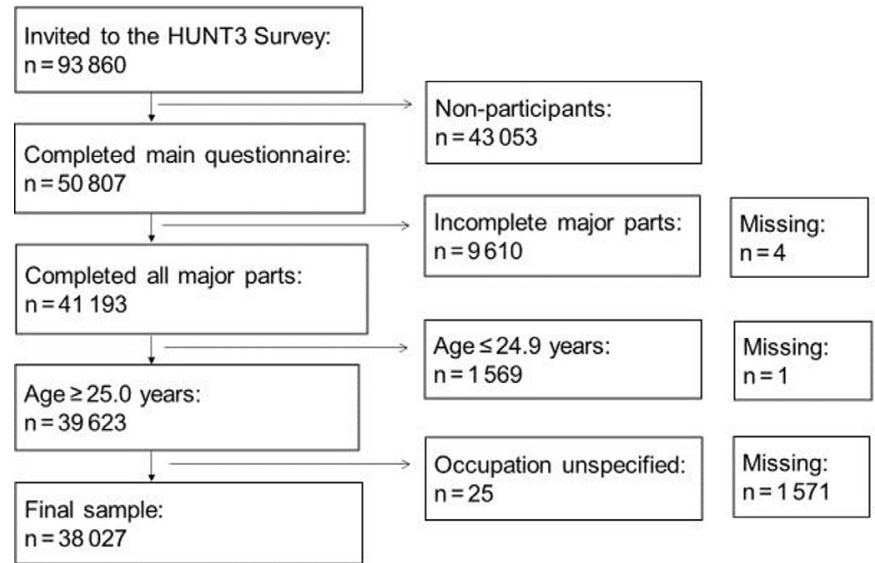

Figure 1 Flowchart for sample selection; inclusion and exclusion criteria and missing data.

the 1980s, and cohort profiles and data collection procedures have been described in detail elsewhere. ${ }^{23}{ }^{24}$ This study is a secondary analysis of data from the HUNT3 Survey (2006-2008), where 93860 citizens were invited to participate. In short, the survey consisted of a main questionnaire received with the invitation by email and handed in when attending a screening station, where participants were interviewed and clinical measurements and biological samples were taken. A second sex-specific and age-specific questionnaire was handed out at the screening station and returned by email.

A total of 50807 individuals (54\% of 93860 invited) completed the main questionnaire, required to be considered an attendant of the HUNT3 Survey. ${ }^{23}$ Sampling is described in figure 1. In this study, 41193 of 50807 participants $(81 \%)$ had data on all major parts of the survey (both questionnaires, interview, measurements and samples) and were designated as respondents. Thus, 9610 were excluded due to incomplete participation, while 4 people missed complete participation data. Under the assumption that young adults may not have obtained their highest level of occupational class at the time of participation, 1569 participants younger than 25 years were excluded, as well as 1 person with missing age data. Occupation data were missing for 1571 respondents, and 25 people were excluded due to unspecified occupation data. Finally, 38027 of 41193 (92\%) respondents were eligible for data analysis, 11204 were non-eligible and 1576 had missing data.

Participation in the HUNT3 Survey varies with socioeconomic position, age and sex. ${ }^{25}$ The distribution of occupational groups among the sample was $24 \%$ (high), 27\% (middle) and 49\% (low) and in non-eligible: $17 \%$ (high), 20\% (middle), 52\% (low) and 11\% (missing). The average (SD) age in the sample was 55 (14) years, in the non-eligible group 44 (18) years and among missing 66 (18) years. Women constituted $55 \%(\mathrm{n}=20813$ of 38 027) of the sample, $51 \%(\mathrm{n}=5662$ of 11203$)$ of the noneligible and $81 \%$ of the missing ( $n=1281$ of 1576). 
Box 1 Conditions grouped by ICD-10 chapter

\section{ICD-10 chapter}

\section{Conditions \\ II Neoplasms}

Cancer

III Blood/blood-forming organs/immune mechanism

Sarcoidosis

IV Endocrine/nutritional/metabolic

Obesity

Hypercholesterolemia

Diabetes

Hypothyroidism

Hyperthyroidism

V Mental/behavioural

Alcohol problem

Depression

Anxiety

Insomnia

Nervous system

Epilepsy

Migraine

Chronic headache, other

VII Eye/adnexa

Cataract

Macula degeneration

Glaucoma

VIII Ear/mastoid

Hearing impairment

IX Circulatory system

Hypertension

Angina pectoris

Myocardial infarction

Heart failure

Other heart disease*

Stroke or brain haemorrhage*

\section{$\mathrm{X}$ Respiratory system}

Chronic bronchitis, emphysema or COPD*

Asthma

\section{Digestive system}

Dental health status

Gastro-oesophageal reflux disease

Irritable bowel syndrome

XII Skin/subcutaneous tissue

Hand eczema

Psoriasis

XIII Musculoskeletal/connective tissue

Rheumatoid arthritis

Osteoarthritis

Ankylosing spondylitis

Fibromyalgia

Osteoporosis

Local musculoskeletal pain/stiffness in:

Neck or upper back or lower back or

shoulder or elbow or hand or

hip or kne or foot/ankle

XIV Genitourinary system

Kidney disease

Urine incontinence

Prostate symptoms

\section{Box 1 Continued}

Menopausal hot flashes

XVIII Symptoms/signs/abnormal clinical/laboratory findings

Nocturia

Chronic widespread pain.

*Exception to single entity

COPD, chronic obstructive pulmonary disease; ICD-10, International

Classification of Diseases, Tenth Revision.

\section{Outcome variable}

Complex multimorbidity was defined as the co-occurrence of three or more chronic conditions affecting three or more different body (organ) systems within one person without defining an index chronic condition', as suggested by previous research. ${ }^{512}$

All conditions possible to generate from the HUNT3 Survey data were included to meet recommendations on deriving the best estimate of prevalence of multimorbidity. ${ }^{12}$ In total, 51 chronic conditions, defined singly as far as original data permitted, were constructed, and details are described in online supplementary appendix A. This list of 51 conditions is more comprehensive and homogeneous than previous operationalisations of multimorbidity in the HUNT3 Survey. ${ }^{21}$

Further, the conditions were grouped according to the International Classification of Diseases,Tenth Revision (ICD-10), in 13 organ-specific chapters and one chapter on symptoms, signs and abnormal clinical and laboratory findings (box 1), using general terms of the conditions in the Norwegian Directorate of eHealth online search engine $^{26}$ on 1 February 2017.

Chapters were counted once if affected by at least one chronic condition, and a summary score of the chapter variables was generated. In this study, complex multimorbidity was defined as having conditions in at least 3 of 14 chapters.

\section{Sociodemographic characteristics}

Occupation data from the HUNT3 Survey were free-text answers to the interview question, "What is/was the title of your main occupation?' Answers were manually categorised corresponding to Standard Classifications of Occupations by Statistics Norway, ${ }^{27}$ which is based on the International Standard Classification of Occupations-88 (ISCO-88). ${ }^{28}$ Socioeconomic position was allocated according to the simplified, 3-class version European Socio-economic Classification (ESeC) scheme. ${ }^{29}$ The simplified scheme is based solely on occupational data, classified according to ISCO$88 .{ }^{28}$ Details are provided in online supplementary appendix B. The intention of the full ESeC scheme is to measure qualitative distinctions between employment relationships and does not reflect a clear hierarchy. ${ }^{29}$ However, income is considered more stable in the salariat class. ${ }^{29}$ In the 3-class version, the salariat class consists of large employers, highergrade and lower-grade professionals, administrative and managerial occupations, and higher-grade technician and 
Table 1 Sex and age distribution by occupational group

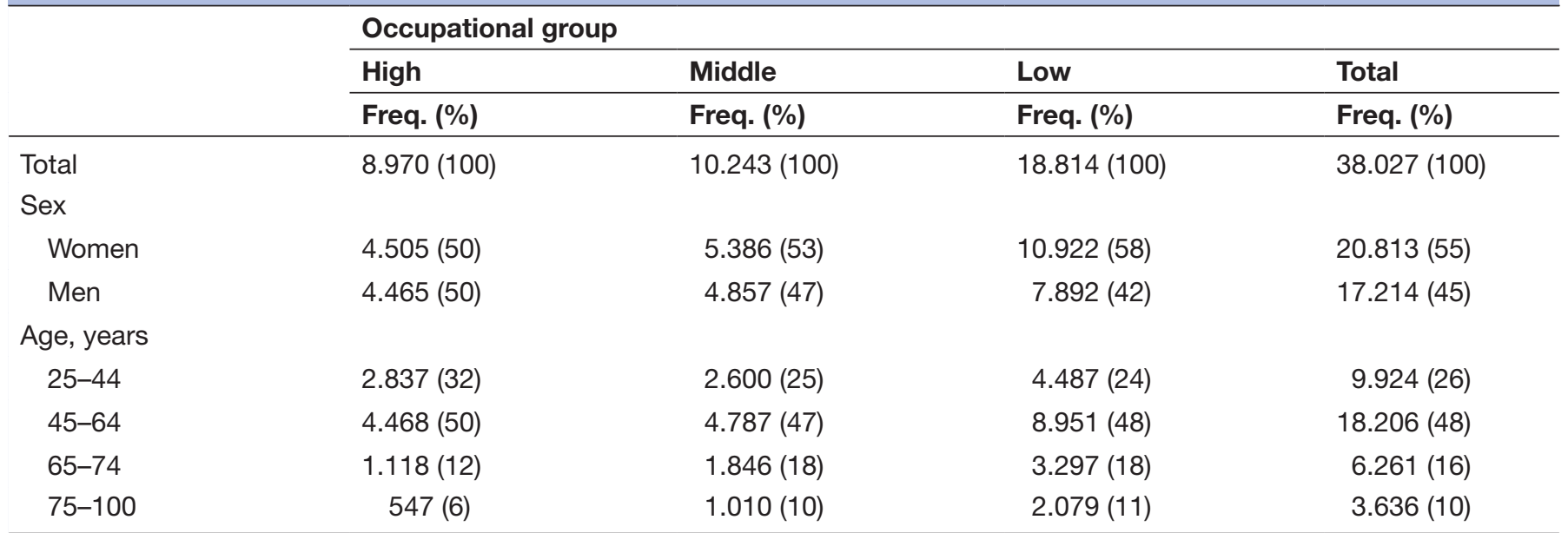

Freq., Frequency.

supervisory occupations. The intermediate class contains small employers, self-employed individuals, and lower-grade supervisory and technician occupations. The working class represents lower-grade service positions, sales and clerical occupations, and lower-grade technical and routine occupations. For practical reasons in this study, the terms high, middle and low occupational group replaced the terms salariat, intermediate and working class, respectively.

In addition, continuous age and categorical sex data, provided by the HUNT Databank, were used in the analyses.

\section{Statistical analysis}

Cross-tables were used to present sociodemographic characteristics of the sample by occupational group (table 1) and by complex multimorbidity, stratified by sex (table 2 ).
Associations between occupational group and complex multimorbidity were analysed using logistic regression. The final models were stratified by sex, included occupational group, continuous age and an interaction term between occupational group and age. Choice of models was guided by likelihood ratio tests.

Since complex multimorbidity was highly prevalent, ORs would deviate from relative risks ${ }^{30}$ and be challenging to interpret. Thus, we used the estimates from the logistic regression models to derive prevalence differences, the difference in mean predicted probability, ${ }^{31}$ and prevalence ratios, the ratio between the mean predicted probabilities, ${ }^{31}$ between occupational groups, while holding other covariates constant. The high occupational group

Table 2 Sociodemographic distribution of complex multimorbidity

\begin{tabular}{|c|c|c|c|c|c|c|}
\hline & \multicolumn{6}{|c|}{ Complex multimorbidity } \\
\hline & \multicolumn{3}{|c|}{ Women } & \multicolumn{3}{|l|}{ Men } \\
\hline & No, n (\%) & Yes, n (\%) & Total, n (\%) & No, n (\%) & Yes, n (\%) & Total, n (\%) \\
\hline Total & $8.505(41)$ & $12.308(59)$ & $20.813(100)$ & $9.137(53)$ & $8.077(47)$ & $17.214(100)$ \\
\hline \multicolumn{7}{|c|}{ Occupational group } \\
\hline High & $2.460(55)$ & $2.045(45)$ & $4.505(100)$ & $2.712(61)$ & $1.753(39)$ & $4.465(100)$ \\
\hline Middle & $2.384(44)$ & $3.002(56)$ & $5.386(100)$ & $2.525(52)$ & $2.332(48)$ & $4.857(100)$ \\
\hline Low & $3.661(34)$ & $7.261(66)$ & $10.922(100)$ & $3.900(49)$ & $3.992(51)$ & $7.892(100)$ \\
\hline \multicolumn{7}{|l|}{ Age, years } \\
\hline 25-44 & $3.859(65)$ & $2.122(35)$ & $5.981(100)$ & $2.958(75)$ & $985(25)$ & $3.943(100)$ \\
\hline $45-64$ & $3.668(37)$ & $6.172(63)$ & $9.840(100)$ & $4.621(55)$ & $3.745(45)$ & $8.366(100)$ \\
\hline $65-74$ & $721(23)$ & $2.447(77)$ & $3.168(100)$ & $1.155(37)$ & $1.938(63)$ & $3.093(100)$ \\
\hline $75-100$ & $257(14)$ & $1.567(86)$ & $1.824(100)$ & $403(22)$ & $1.409(78)$ & $1.812(100)$ \\
\hline Mean (SD) & $48(13)$ & 59.(14) & $54(14)$ & $52(13)$ & $62(13)$ & $56(14)$ \\
\hline
\end{tabular}


Table 3 Prevalence ratios (PRs) and prevalence differences (PDs) with 95\% Cls in complex multimorbidity between occupational groups, stratified by sex

\begin{tabular}{|c|c|c|c|c|c|}
\hline \multirow[b]{2}{*}{ Age, years } & \multirow{2}{*}{$\begin{array}{l}\text { Occupational } \\
\text { group }\end{array}$} & \multicolumn{2}{|l|}{ Women } & \multicolumn{2}{|l|}{ Men } \\
\hline & & PR $(95 \% \mathrm{Cl})$ & PD $(95 \% \mathrm{Cl})$ & PR $(95 \% \mathrm{Cl})$ & PD $(95 \% \mathrm{Cl})$ \\
\hline \multirow[t]{2}{*}{30} & High & 1.00 (ref.) & 0.00 (ref.) & 1.00 (ref.) & 0.00 (ref.) \\
\hline & Low & 2.06 (1.84 to 2.32 ) & 0.19 (0.16 to 0.21$)$ & 1.92 (1.63 to 2.26$)$ & 0.10 (0.08 to 0.13$)$ \\
\hline \multirow[t]{2}{*}{55} & High & 1.00 (ref.) & 0.00 (ref.) & 1.00 (ref.) & 0.00 (ref.) \\
\hline & Low & $1.22(1.18$ to 1.26$)$ & $0.12(0.10$ to 0.14$)$ & 1.35 (1.28 to 1.41$)$ & $0.13(0.11$ to 0.15$)$ \\
\hline \multirow[t]{3}{*}{75} & High & 1.00 (ref.) & 0.00 (ref.) & 1.00 (ref.) & 0.00 (ref.) \\
\hline & Middle & 0.98 (0.95 to 1.02$)$ & $-0.01(-0.04$ to 0.02$)$ & 1.07 (1.02 to 1.12$)$ & 0.05 (0.02 to 0.08$)$ \\
\hline & Low & $1.02(0.99$ to 1.05$)$ & $0.02(-0.01$ to 0.04$)$ & $1.10(1.06$ to 1.15$)$ & 0.07 (0.04 to 0.10$)$ \\
\hline 90 & High & 1.00 (ref.) & 0.00 (ref.) & 1.00 (ref.) & 0.00 (ref.) \\
\hline
\end{tabular}

was chosen as the reference group. Prevalence differences and prevalence ratios were calculated in 5-year intervals from 25 to 100 years, with $95 \%$ CIs (online supplementary appendix C). Results for the ages 30, 55, 75 and 90 years are presented in table 3 to represent adult, middle aged, aged and oldest old in the sample.

To visualise the differential association between age and complex multimorbidity in each occupational group, we specified separate models using restricted cubic splines and graphed the findings from each model into a common plot for each sex.

Sensitivity analysis was performed to investigate if the number and types of conditions showed a similar pattern with respect to the overall prevalence as well as differences between occupational groups (online supplementary appendix D). The alternative complex multimorbidity measure was derived from data in the main questionnaire only (22 conditions, grouped in 12 ICD-10 chapters).

Complete case analysis was performed, and StataIC 15.1 was used to analyse the data (StataCorp. 2017. Stata Statistical Software: Release 15. College Station, TX: StataCorp LLG).

\section{Patient and public involvement}

There was a broad participant, patient and stakeholder involvement during the planning of the HUNT3 Survey. Data collection was performed in 2006-2008. Complex multimorbidity is a universal subject, not represented by any particular patient group, and thus no patient or public representative was involved in the design of this secondary analysis study.

\section{RESULTS}

Thirty-eight thousand twenty-seven individuals, aged 25-100 years, 55\% women $(n=20813)$, who had completed all major parts of the HUNT3 Survey and had a classifiable occupation comprised the eligible sample, as figure 1 depicts. table 1 presents further sociodemographic characteristics.

Nearly half the sample $(49 \%$; $n=18814$ of 38027 ; of which $58 \%$ were women, $\mathrm{n}=10922$ ) was allocated in the low occupational group. In absolute numbers, the low occupational group was the largest socioeconomic category in both sexes and all age groups. The proportion of individuals aged 25-44 years decreased from 32\% in the high occupational group $(n=2837)$ to $24 \%$ in the low occupational group $(n=4487)$, while the proportion of individuals aged 75 to 100 years increased from $6 \%(n=547)$ to $11 \%(n=2079)$. Participants aged 45 to 64 years were the largest age group in total and in all occupational groups (high, $\mathrm{n}=4468$; middle, $\mathrm{n}=4787$; low, $\mathrm{n}=8951$ ).

Overall, a majority ( $54 \%$; $=20385$ of 38 027) of the sample met the criteria for having complex multimorbidity, including $59 \%$ of women $(n=12$ 308) and $47 \%$ of men ( $n=8077$; table 2$)$. The percentages increased from high to low occupational group in women from $45 \%(\mathrm{n}=2045)$ to $66 \%(\mathrm{n}=7261)$ and in men from $39 \%$ $(\mathrm{n}=1753)$ to $51 \% \quad(\mathrm{n}=3992)$. The proportions further increased by age, from $35 \%(n=2122)$ of women aged 25 to 44 years to $86 \%(n=1567)$ of women aged 75 to 100 years. In men, the increase was from $25 \%(n=985)$ to $78 \%$ $(n=1409)$ in the same age groups. In absolute numbers, most people classified as having complex multimorbidity were aged 45 to 64 years (women, $n=6172$; men, $n=3745$ ).

Table 3 shows prevalence ratios and prevalence differences between the occupational groups after adjusting for age and occupation-age interaction and thus presented at ages 30, 55, 75 and 90 years. Prevalence differences for complex multimorbidity between high and low occupational groups varied; at 30 years, 19 (16 to 21) percentage points (pp) in women and 10 (8 to 13) pp in men; at 55 years, 12 (10 to 14 ) pp in women and 13 (11 to 15) pp in 


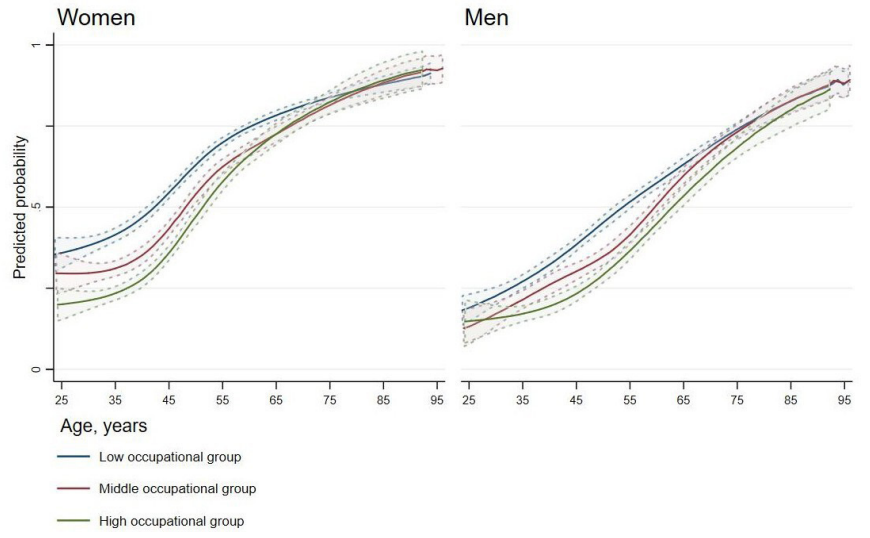

Figure 2 Estimated prevalence of complex multimorbidity with $95 \%$ Cls by age and occupational group for women and men.

men; at 75 years, 2 ( -1 to 4 ) pp in women and 7 (4 to 10 ) $\mathrm{pp}$ in men; and at 90 years, -1 ( -3 to 1 ) pp in women and $2(-1$ to 5$)$ in men. Compared with the high occupational group, the prevalence ratios for the low occupational group for complex multimorbidity were at 30 years, 2.06 ( 1.84 to 2.32 ) in women and 1.92 (1.63 to 2.26) in men; at 55 years, 1.22 (1.18 to 1.26$)$ in women and 1.35 (1.28 to 1.41$)$ in men; at 75 years, 1.02 (0.99 to 1.05$)$ in women and 1.10 (1.06 to 1.15$)$ in men; and at 90 years, 0.99 (0.97 to 1.01 ) in women and 1.03 (0.99 to 1.07 ) in men.

In the sensitivity analyses where the complex multimorbidity measure was derived from fewer conditions (22 vs 51) and ICD-10 chapters (12 vs 14), the total prevalence was $15 \%$ ( $n=5836$ of 38027 , online supplementary appendix D). Proportions were greater in women, higher age and the low occupational group. Compared with the results from the main analysis, prevalence differences between high and low occupational groups were smaller in women at all ages and in men at age 30 years and 55 years, while prevalence ratios were greater in men at all ages and in women aged 30 and 55 years.

Figure 2 depicts estimated prevalence of complex multimorbidity by occupational group and sex in individuals aged 25 to 100 years. In all occupational groups in both sexes, the predicted prevalence increased with age throughout the age span. Further, estimated prevalence differed between the occupational groups in women until age 75 years and in men until age 90 years. Women had a consistently higher prevalence for complex multimorbidity than men.

\section{DISCUSSION}

\section{Main results}

More than half $(54 \%)$ of this total county adult population sample were identified with complex multimorbidity, measured as occurrence of chronic conditions in minimum three separate organ systems. Prevalence of complex multimorbidity was common from early adulthood, increased with age and was higher in women and in the low occupational group. Occupational group prevalence differences and ratios in complex multimorbidity were diminishing in women, while still present in men, at age 75 years.

\section{Comparison with existing literature}

Few, if any, studies (to our knowledge) have investigated the prevalence and determinants of complex multimorbidity in a general population. The findings are in keeping with known determinants of lower social position, female sex and higher age for multimorbidity in both general population ${ }^{19}$ and primary care studies. ${ }^{314} 16$ An Australian study using a comparable operationalisation of complex multimorbidity identified nearly $25 \%$ of patients in general practice with complex multimorbidity and estimated a national prevalence of $17 \% .{ }^{32}$ However, higher prevalence findings from our predominantly selfreported data are compatible with studies comparing prevalence estimates from self-reports and health record data. ${ }^{33}$ In absolute numbers, the incidence of individuals identified with the stricter measure of complex multimorbidity is still highest among the group younger than 64 years, as has been shown for multimorbidity. ${ }^{16} 1935$ The sensitivity analysis confirms how number and types of conditions influence prevalence ${ }^{1215}$ and effect estimates of age, sex and socioeconomic position. ${ }^{36}$

\section{Mechanisms to explain findings}

The association between lower socioeconomic position and poor health is well established. In general, unequal distribution of income, power and wealth is understood to be socially determined fundamental causes that impact conditions of everyday life and result in social health inequalities. ${ }^{17}$ In Nordic countries assumed to be egalitarian and offering universal healthcare, social health inequalities still exist. ${ }^{18}$ Theories put forward are the survival of individuals with greater frailty, who are more likely to obtain a lower social position. ${ }^{37}$ The gap in health is also explained by overall morbidity and mortality decreasing faster among the higher than the lower socioeconomic groups. ${ }^{37}$

In this study, occupational group serves as the proxy variable for socioeconomic position. Occupation may affect health outcomes through universal and specific mechanisms. In general, the higher occupational groups will have more secure and higher income, ${ }^{29} 38$ as well as advantageous social networks. ${ }^{38}$ In particular, jobs vary in psychosocial factors, such as stress, control and autonomy and biological factors, such as physical demands or harmful and hazardous work environments. ${ }^{38}$ Overall, the higher occupational groups have greater autonomy and control, ${ }^{29}$ while lower occupational groups are more exposed to malign work factors. ${ }^{17}$ Generations may have different associations between a profession and health outcomes, ${ }^{38}$ as occupations, tasks and exposures shift over time.

The bidirectional relationship between health and occupation ${ }^{20}$ may partly explain the larger prevalence differences and ratios between low and high occupational 
groups in the younger age categories. Higher rates of multimorbidity in young individuals in lower socioeconomic positions may also be explained by detection bias $^{35}$ in which the initiation of therapy and healthcare follow-up increase the likelihood of diagnosing more conditions. Diminishing occupational ratios and differences among the oldest may be explained by the higher overall prevalence of complex multimorbidity ${ }^{39}$ and also survival bias, whereby the individuals with greatest fragility have already died. While probability of complex multimorbidity increases with age, the age distribution results in a higher number of cases occurring in those younger than 64 years.

\section{Strengths and limitations}

Strength of this study is the estimation of prevalence of complex multimorbidity from a general population survey, the most common study design in multimorbidity studies. ${ }^{40}$ A vast number of self-reported conditions are included, almost exclusively diagnoses and symptoms. ${ }^{40}$ Self-report is considered a valid approach when studying large samples. ${ }^{15}$ Furthermore, using all available data will produce the most proper prevalence estimates ${ }^{12}$ which in this study is demonstrated by the sensitivity analysis and which seems necessary to detect occupational differences in younger age groups. The sensitivity analyses confirm that the spectrum of conditions included may affect associations with socioeconomic position, age and sex. ${ }^{36}$

Our operationalisation of complex multimorbidity makes the prevalence estimates comparable with other studies categorising conditions by any organ-based system. ${ }^{12}$ The occurrence of conditions in separate organ, and number of organ systems, could have been explored as a continuous measure with assumed increasing severity; however, this was beyond the scope of this study.

The allocation of occupations in the ESeC also makes international comparison of social gradients possible. ${ }^{29}$ We presented absolute and relative differences in compliance with recommendations on measurements of socioeconomic inequalities in health. ${ }^{41}$ Results are further stratified by age and sex, which are stated as minimum requirements for proper reporting of multimorbidity. ${ }^{14}$

A number of limitations should be noted. Our study is based on data collected for a general health survey, and this limits data on conditions included in the complex multimorbidity measure. In particular, we did not have explicit information on chronicity for a majority of the conditions. Thus, the prevalence of complex multimorbidity may be overestimated.

Socioeconomic position was explored using only occupation, and while social health inequalities will be detected, ${ }^{20}$ socioeconomic measures are not interchangeable. $^{2042}$ Different measures of socioeconomic position will act through varying mechanisms and may associate distinctively with health outcomes. ${ }^{20}{ }^{42}$ Participants in the HUNT3 Survey reported their main occupation, while current or longest lasting occupation is more often studied. ${ }^{38}$ Younger subjects may be misclassified in lower socioeconomic position, which may underestimate the occupational differences in health in this age group, whereas reverse causation, whereby prior health status determines job opportunities, is unavoidable and will increase detected differences. This study excludes those never having worked, which will underestimate social gradients in complex comorbidity. ${ }^{43}$ Further, individuals with data missing due to unclassifiable occupation, a circumstance more common in elderly women than other participants, were excluded. Occupational data may misrepresent present social context ${ }^{38}$ and thereby underestimate social inequalities. It would have been favourable if the study had included education, income or household indicators for socioeconomic position.

Participation in the HUNT3 Survey varied by age, sex, socioeconomic position and pattern of morbidity. ${ }^{25}$ This may weaken the effect estimates of the determinants to complex multimorbidity. A healthy elders bias is likely, since participation required attendance at a screening station. ${ }^{23}$ Overall, prevalence of individual conditions has shown only slight differences between participants and non-participants. ${ }^{25}$ The HUNT Study is considered fairly representative for Norway, ${ }^{24}$ and the health development in the material follows western high-income country trends closely. ${ }^{44-46}$

\section{Implications for clinical practice and policy makers}

Our study confirms that complex multimorbidity, a suggested measure to identify multimorbid individuals with high need for coordinated multidisciplinary care, ${ }^{12}$ is highly prevalent in the general population, where social differences are evident from young to old adulthood. This is in line with international studies, and at policy level, an emphasis on public health intervention to prevent complex multimorbidity and social differences seems necessary. As proposed elsewhere, this will likely require a proportionate universalism life-cycle approach. ${ }^{47}$ To improve and secure healthcare for this large patient group, clinical guidelines and the organisation of healthcare are suggested to adapt to a personcentred, generalist approach. ${ }^{5} 10$

\section{Future research}

Complex multimorbidity is common in this general population sample, with a clear social gradient throughout adulthood. Careful interpretation is necessary, since there are possible biases in measures of multimorbidity and occupation. However, the HUNT3 Survey data cover a broad spectrum of conditions and give a unique opportunity to create several measures of multimorbidity in the same sample, with directly comparable prevalence estimates and gradients. On this background, we recommend exploring alternative measures suggested to detect individuals with high needs and multimorbidity and investigate differences in patterns and consequences of such measures by social health determinants. Since multimorbidity is the norm and represents a large challenge to healthcare across levels, research on overall healthcare 
utilisation and organisation should be a priority, as well as studying competing measures as prognostic factors for mortality. Studies on social differences in the use of healthcare may identify vulnerable subgroups, where any specific organisation of treatment later on could be evaluated.

\section{CONCLUSION}

Complex multimorbidity, defined as occurrence of chronic conditions in three separate organ systems, is common, and occupational differences exist throughout adulthood in both sexes. The magnitude of complex multimorbidity in all age groups implies the need for public health management to universally improve, targeted proportionate to need and disadvantage in subpopulations, social health determinants throughout the lifespan. Complex multimorbidity, indicating the accumulation of conditions of different aetiology requiring coordinated multidisciplinary care, should inspire health caregivers, healthcare organisations, educational institutions and researchers to take on a generalist and person-centred focus. Studying alternative multimorbidity measures, including healthcare utilisation and mortality according to social background, as well as multimorbidity management, should be prioritised in future research.

\section{Author affiliations}

${ }^{1}$ HUNT Research Centre, Department of Public Health and Nursing, Faculty of Medicine and Health Science, Norwegian University of Science and Technology, Levanger, Trøndelag, Norway

${ }^{2}$ Psychiatric Department, Levanger Hospital, Nord-Trøndelag Hospital Trust, Levanger, Trøndelag, Norway

${ }^{3}$ Faculty of Nursing and Health Sciences, Nord Universitet - Levanger Campus, Levanger, Norway

${ }^{4}$ Department of Mental Health, Faculty of Medicine and Health Sciences, Norwegian University of Science and Technology, Trondheim, Trøndelag, Norway

${ }^{5}$ Department of Public Health and Nursing, Faculty of Medicine and Health Sciences, Norwegian University of Science and Technology, Trondheim, Trøndelag, Norway ${ }^{6}$ Academic Unit of General Practice, Australian National University Medical School, Australian National University, Canberra, Australian Capital Territory, Australia ${ }^{7}$ Levanger Hospital, Nord-Trøndelag Hospital Trust, Levanger, Trøndelag, Norway

\section{Twitter Kristin Hestmann Vinjerui @KHVinjerui and Steinar Krokstad @steinak}

Acknowledgements L Getz, J Sigurdsson and C Harrison for through discussions on measures of multimorbidity. E Solheim for guidance in the use of the European Socio-economic Classification scheme.MS Newman for writing assistance. The Nord-Trøndelag Health Study (The HUNT Study) is a collaboration between HUNT Research Centre (Faculty of Medicine and Health Sciences, Norwegian University of Science and Technology (NTNU), Nord-Trøndelag County Council, Central Norway Regional Health Authority, and the Norwegian Institute of Public Health.)

Contributors KHV, ERS, SK and JHB conceptualised the study and KHV, ERS, SK, JHB, OB and KAD contributed to its design. KHV has analysed the data under supervision of ERS, and KHV, ERS, SK, JHB, OB and KAD have contributed to interpreting the data. KHV wrote the original draft, which has been revised critically by ERS, SK, JHB, OB and KAD. KHV, ERS, SK, JHB, OB and KAD have read and approved the final version of the manuscript to be published and agree to be accountable for all aspects of the work in ensuring that questions related to the accuracy or integrity of any part of the work are appropriately investigated and resolved.

Funding This study was funded by the Faculty of Medicine and Health Science at the Norwegian University of Science and Technology (NTNU) through the PhD program in Behavior and Health (KHV). NTNU has partly funded the HUNT3 Survey and have funded open access for this article. The Liaison Committee for Education, Research and Innovation in Central Norway (17/38297) supported a research stay at the Australian National University, Canberra. The funding sources have had no role in conceptualisation this study, its design and methods, analysis and interpretation of data, writing of the article or the decision to submit the article for publication.

Competing interests None declared.

Patient and public involvement Patients and/or the public were not involved in the design, or conduct, or reporting, or dissemination plans of this research.

Patient consent for publication Not required.

Ethics approval The Regional Committee for Medical and Health Research Ethics in Norway approved the current study (project no. 2014/2265).

Provenance and peer review Not commissioned; externally peer reviewed.

Data availability statement Data are available upon reasonable request. To protect participants' privacy, HUNT Research Centre aims to limit storage of data outside HUNT Databank and cannot deposit data in open repositories. HUNT Databank has precise information on all data exported to different projects and are able to reproduce these on request. There are no restrictions regarding data export given approval of applications to HUNT Research Centre. For more information see: http://www.ntnu.edu/hunt/data.

Open access This is an open access article distributed in accordance with the Creative Commons Attribution Non Commercial (CC BY-NC 4.0) license, which permits others to distribute, remix, adapt, build upon this work non-commercially, and license their derivative works on different terms, provided the original work is properly cited, appropriate credit is given, any changes made indicated, and the use is non-commercial. See: http://creativecommons.org/licenses/by-nc/4.0/.

ORCID iD

Kristin Hestmann Vinjerui http://orcid.org/0000-0003-1828-6943

\section{REFERENCES}

1 Nicholson K, Makovski TT, Griffith LE, et al. Multimorbidity and comorbidity revisited: Refining the concepts for international health research. J Clin Epidemiol 2019;105:142-6.

2 van Oostrom SH, Gijsen R, Stirbu I, et al. Time trends in prevalence of chronic diseases and multimorbidity not only due to aging: data from general practices and health surveys. PLoS One 2016;11:e0160264.

3 Uijen AA, van de Lisdonk EH. Multimorbidity in primary care: prevalence and trend over the last 20 years. Eur J Gen Pract 2008;14:28-32.

4 Coventry PA, Fisher L, Kenning C, et al. Capacity, responsibility, and motivation: a critical qualitative evaluation of patient and practitioner views about barriers to self-management in people with multimorbidity. BMC Health Serv Res 2014;14:536.

5 Wallace E, Salisbury C, Guthrie B, et al. Managing patients with multimorbidity in primary care. BMJ 2015;350:h176.

6 Søndergaard E, Willadsen TG, Guassora AD, et al. Problems and challenges in relation to the treatment of patients with multimorbidity: general practitioners' views and attitudes. Scand J Prim Health Care 2015;33:121-6.

7 Guthrie B, Payne K, Alderson P, et al. Adapting clinical guidelines to take account of multimorbidity. BMJ 2012;345:e6341.

8 Glynn LG, Valderas JM, Healy P, et al. The prevalence of multimorbidity in primary care and its effect on health care utilization and cost. Fam Pract 2011;28:516-23.

9 Agborsangaya CB, Lau D, Lahtinen M, et al. Health-Related quality of life and healthcare utilization in multimorbidity: results of a crosssectional survey. Qual Life Res 2013;22:791-9.

10 National Institute for Health and Care Excellence. Multimorbidity: clinical assessment and management. NICE guideline [NG56]. National Institute for Health and Care Excellence, 2016. Available: https://www.nice.org.uk/guidance/ng56

11 Palmer K, Marengoni A, Forjaz MJ, et al. Multimorbidity care model: recommendations from the consensus meeting of the joint action on chronic diseases and promoting healthy ageing across the life cycle (JA-CHRODIS). Health Policy 2018;122:4-11.

12 Harrison C, Britt H, Miller G, et al. Examining different measures of multimorbidity, using a large prospective cross-sectional study in Australian general practice. BMJ Open 2014;4:e004694.

13 Schaink AK, Kuluski K, Lyons RF, et al. A scoping review and thematic classification of patient complexity: offering a unifying framework. J Comorb 2012;2:1-9. 
14 Violan C, Foguet-Boreu Q, Flores-Mateo G, et al. Prevalence, determinants and patterns of multimorbidity in primary care: a systematic review of observational studies. PLoS One 2014:9:e102149.

15 Fortin M, Stewart M, Poitras M-E, et al. A systematic review of prevalence studies on multimorbidity: toward a more uniform methodology. Ann Fam Med 2012;10:142-51.

16 Barnett K, Mercer SW, Norbury M, et al. Epidemiology of multimorbidity and implications for health care, research, and medical education: a cross-sectional study. Lancet 2012;380:37-43.

17 Commission on Social Determinants of Health, In Marmot M, ed. Closing the gap in a generation: health equity through action on the social determinants of health: final report of the Commission on social determinants of health. Geneva, 2008: 256.

18 Mackenbach JP, Stirbu I, Roskam A-JR, et al. Socioeconomic inequalities in health in 22 European countries. N Engl J Med 2008;358:2468-81.

19 Agborsangaya CB, Lau D, Lahtinen M, et al. Multimorbidity prevalence and patterns across socioeconomic determinants: a cross-sectional survey. BMC Public Health 2012;12:201.

20 Galobardes B, Lynch J, Smith GD. Measuring socioeconomic position in health research. Br Med Bull 2007;81:21-37.

21 Tomasdottir MO, Getz L, Sigurdsson JA, et al. Co- and multimorbidity patterns in an unselected Norwegian population: cross-sectional analysis based on the HUNT study and theoretical reflections concerning basic medical models. Eur J Pers Cent Healthc 2014;2:335-45.

22 von Elm E, Altman DG, Egger M, et al. The strengthening the reporting of observational studies in epidemiology (STROBE) statement: guidelines for reporting observational studies. Int J Surg 2014;12:1495-9.

23 Krokstad S, Langhammer A, Hveem K, et al. Cohort profile: the HUNT study, Norway. Int J Epidemiol 2013;42:968-77.

24 Holmen J, Midthjell K, $\varnothing \mathrm{K}$, et al. The Nord-Trøndelag health study 1995-97 (Hunt 2): objectives, contents, methods and participation. Norsk epidemiologi 2003;13:19-32.

25 Langhammer A, Krokstad S, Romundstad P, et al. The HUNT study: participation is associated with survival and depends on socioeconomic status, diseases and symptoms. BMC Med Res Methodol 2012;12:143.

26 Direktoratet for e-helse. FinnKode - Helsedirektoratet medisinske kodeverk, 2017. Available: https://finnkode.ehelse.no/\#icd10/0/0/ $0 /-1$

27 Norway S. Standard classification of occupations. Statistics Norway: Oslo/Kongsvinger, 1998.

28 International Labour Organization (ILO). The International Standard Classification of Occupations, ISCO-88 [Webpage], 1988. Available: https://www.ilo.org/public/english/bureau/stat/isco/isco88/index.htm [Accessed 18 Sep 2004].

29 Rose D, Harrison E. The European socio-economic classification: a new social class schema for comparative European research. Eur Soc 2007;9:459-90.

30 Sedgwick P. Relative risks versus odds ratios. BMJ 2014;348:g1407-g.

31 Norton EC, Miller MM, Kleinman LC. Computing adjusted risk ratios and risk differences in Stata. Stata J 2013;13:492-509.
32 Harrison C, Henderson J, Miller G, et al. The prevalence of complex multimorbidity in Australia. Aust N Z J Public Health 2016;40:239-44.

33 Cricelli C, Mazzaglia G, Samani F, et al. Prevalence estimates for chronic diseases in Italy: exploring the differences between self-report and primary care databases. J Public Health Med 2003;25:254-7.

34 Fortin M, Haggerty J, Sanche S, et al. Self-Reported versus health administrative data: implications for assessing chronic illness burden in populations. A cross-sectional study. CMAJ Open 2017;5:E729-33.

35 van den Akker M, Buntinx F, Metsemakers JF, et al. Multimorbidity in general practice: prevalence, incidence, and determinants of co-occurring chronic and recurrent diseases. J Clin Epidemiol 1998;51:367-75.

36 Schäfer I, Hansen H, Schön G, et al. The influence of age, gender and socio-economic status on multimorbidity patterns in primary care. first results from the multicare cohort study. BMC Health Serv Res 2012;12:89.

37 Huijts T, Eikemo TA. Causality, social selectivity or artefacts? why socioeconomic inequalities in health are not smallest in the Nordic countries. Eur J Public Health 2009;19:452-3.

38 Galobardes B, Shaw M, Lawlor DA, et al. Indicators of socioeconomic position (Part 1). J Epidemiol Community Health 2006;60:7-12.

39 Scanlan JP, Editorial G. Guest editorial. CHANCE 2006;19:47-51.

40 Willadsen TG, Bebe A, Køster-Rasmussen R, et al. The role of diseases, risk factors and symptoms in the definition of multimorbidity - a systematic review. Scand J Prim Health Care 2016;34:112-21.

41 Mackenbach JP, Kunst AE. Measuring the magnitude of socioeconomic inequalities in health: an overview of available measures illustrated with two examples from Europe. Soc Sci Med 1997;44:757-71.

42 Braveman PA, Cubbin C, Egerter S, et al. Socioeconomic status in health research: one size does not fit all. JAMA 2005;294:2879-88.

43 Martikainen $\mathrm{P}$, Valkonen T. Bias related to the exclusion of the economically inactive in studies on social class differences in mortality. Int J Epidemiol 1999;28:899-904.

44 NCD Risk Factor Collaboration (NCD-RisC). Rising rural body-mass index is the main driver of the global obesity epidemic in adults. Nature 2019:569:260-4.

45 NCD Risk Factor Collaboration (NCD-RisC). Worldwide trends in blood pressure from 1975 to 2015: a pooled analysis of 1479 population-based measurement studies with 19.1 million participants. Lancet 2017;389:37-55.

46 NCD Risk Factor Collaboration (NCD-RisC). Worldwide trends in body-mass index, underweight, overweight, and obesity from 1975 to 2016: a pooled analysis of 2416 population-based measurement studies in 128.9 million children, adolescents, and adults. Lancet 2017;390:2627-42

47 Marmot M, Goldblatt P, Allen J, et al. Fair Society, healthy lives: the Marmot review 2010:242.

48 World Health Organization. Multimorbidity: technical series on safer primary care. Geneva: World Health Organization, 2016: 4-5. 